\title{
Cutset Width and Spacing for Reduced Cutset Coding of Markov Random Fields
}

\author{
Matthew G. Reyes* \\ *self-employed \\ mgreyes@umich.edu
}

\author{
David L. Neuhoff ${ }^{\dagger}$ \\ †EECS Dept., University of Michigan \\ neuhoff@umich.edu
}

\begin{abstract}
In this paper we explore tradeoffs, regarding coding performance, between the thickness and spacing of the cutset used in Reduced Cutset Coding (RCC) of a Markov random field image model [10]. Considering MRF models on a square lattice of sites, we show that under a stationarity condition, increasing the thickness of the cutset reduces coding rate for the cutset, increasing the spacing between components of the cutset increases the coding rate of the non-cutset pixels, though the coding rate of the latter is always strictly less than that of the former. We show that the redundancy of RCC can be decomposed into two terms, a correlation redundancy due to coding the components of the cutset independently, and a distribution redundancy due to coding the cutset as a reduced MRF. We provide analysis of these two sources of redundancy. We present results from numerical simulations with a homogeneous Ising model that bear out the analytical results. We also present a consistent estimation algorithm for the moment-matching reduced MRF for the cutset $U$.
\end{abstract}

\section{Introduction}

A Markov random field (MRF) $X=\left\{X_{i}: i \in V\right\}$ is a collection of random variables on an undirected graph graph $G=(V, E)$, where the nodes 11 in $V$ are the random variable indices and the edges in $E$ represent direct dependencies between the random variables [14], and is often proposed as a model for many sources of data, such as images. A family of MRFs on a graph $G$ is defined by a vector statistic $t$ having a component for each edge and each node. An individual MRF within this family is indicated by an exponential parameter vector $\theta$ whose components correspond to the components of $t$. Since there has been relatively little development of algorithms or theory for the compression of MRFs [2,7,11,13, we feel that this is an important problem to consider. In this paper we explore design tradeoffs of the lossless Reduced Cutset Coding method introduced in [10].

Reduced Cutset Coding (RCC) is a two-stage algorithm for lossless compression of an MRF defined on an intractable graph, where tractability is with respect to Belief Propagation (BP) [10,11, 14]. The method consists, first, of suboptimal lossless encoding of a cutset $U \subset V$, chosen such that the subgraphs $G_{U}$ and $G_{W}$ induced by $U$ and $W=V \backslash U$, respectively, are tractable. The components of $X_{U}$ are encoded with Arithmetic Coding (AC) using BP to compute a reduced MRF coding distribution. A reduced MRF for $X_{U}$ is an MRF on the subgraph $G_{U}$ induced by $U$, with the statistic

An abbreviated version of this paper has been submitted to ISIT 2016.

${ }^{1}$ We use the terms nodes, sites and pixels interchangeably. 
$t$ limited to $U$, and a possibly different exponential parameter vector $\tilde{\theta}_{U}$. Secondly, conditioned on the encoded cutset $X_{U}$, the component subsets of the remaining variables $X_{W}$ are encoded conditioned on their respective boundaries, again using $\mathrm{AC}$, with $\mathrm{BP}$ used to compute the true conditional coding distributions of the variables in $X_{W}$ with respect to the original MRF.

The rate of this scheme can be expressed as

$$
R=\frac{|U|}{|V|} R_{U}+\frac{|W|}{|V|} R_{W}
$$

where $R_{U}$ is the rate in bits per pixel for the cutset $U$, and likewise $R_{W}$ for the remainder $W$. Because $G_{W}$ is tractable for BP, the conditional coding distributions for the components of $X_{W}$ can be exactly computed. Thus AC will encode each component on average at its conditional entropy plus an overhead of one or two bits [15]. Since we have in mind the components of $W$ having many pixels, the rate $R_{W}$ is well-approximated by $\frac{1}{|W|} H\left(X_{W} \mid X_{U}\right)$, the ideal coding rate for $X_{W}$ given $X_{U}$. Similarly, since $U$ is tractable for BP, the reduced MRF coding distribution can be computed exactly, and $R_{U}$ is well-approximated by the (normalized) cross entropy $\frac{1}{|U|} H\left(X_{U} \| \tilde{X}_{U}\right)$ between the marginal distribution for $X_{U}$ and the reduced MRF distribution for the same variables, which we denote $\tilde{X}_{U}$, and which equals the entropy $\frac{1}{|U|} H\left(X_{U}\right)$ of $X_{U}$ plus the divergence $\frac{1}{|U|} D\left(X_{U}|| \tilde{X}_{U}\right)$ between the true and reduced MRF distributions for $X_{U}$.

It follows that the rate of this scheme exceeds the rate of an optimal code, which is

$$
\frac{1}{|V|} H\left(X_{U}, X_{W}\right)=\frac{|U|}{|V|} \frac{1}{|U|} H\left(X_{U}\right)+\frac{|W|}{|V|} \frac{1}{|W|} H\left(X_{W} \mid X_{U}\right)
$$

by the divergence $\frac{1}{|V|} D\left(X_{U} \| \tilde{X}_{U}\right)$. For a given cutset $U$, this divergence is minimized by choosing the parameter vector $\tilde{\theta}_{U}$ to be that which causes the mean of the statistic $t_{U}$ of the reduced MRF $\tilde{X}_{U}$ to be the same as the mean of $t_{U}$ on the marginal $X_{U}$ of the original MRF $X[1,10,14]$. This is called the moment-matching parameter and denoted $\theta_{U}^{*}$. In Section 4 we present a consistent algorithm for estimating $\theta_{U}^{*}$ for a tractable subset $U$, and as such, for the rest of this paper we let $\tilde{X}_{U}$ denote this moment-matching reduced MRF. Even when divergence is minimized, one normally expects $\frac{1}{|U|} H\left(X_{U}\right)$ to be larger than $\frac{1}{|W|} H\left(X_{W} \mid X_{U}\right)$.

In the present paper we consider an $\mathrm{MRF}$ on an $M \times N$ rectangular lattice of sites. The statistic $t$ as well as the parameter $\theta$ are both row-invariant, and the image height $M$ is assumed to be very large, so that the sequences of rows of the image are assumed to form a stationary process. The cutset $U$ consists of $k+1$ evenly spaced $n_{L} \times N$ rectangular regions $L_{1}, \ldots, L_{k+1}$, referred to as lines, so that the $k$ components of $G_{W}$ are themselves $n_{S} \times N$ rectangular regions $S_{1}, \ldots, S_{k}$, referred to as strips. This is an extension of the RCC method of [10], [11], which restricted $n_{L}$ to be $12^{2}$ Here, $M=k n_{S}+(k+1) n_{L}$, so that lines and strips alternate, beginning with a line and ending with a line. This class of cutsets was chosen to simplify both

\footnotetext{
${ }^{2}$ Even though now $n_{L}$ can be larger than one, we continue to use the nomenclature of lines.
} 
the algorithm and the analysis. For example, the lines (strips) can be transformed into a simple chain graph by grouping the pixels in each column of a line (strip) into one superpixel. If $n_{L}$ and $n_{S}$ are both moderate, for instance at most 10 , then BP can be used to perform exact inference efficiently.

An interesting question is how the cutset parameters $n_{L}$ and $n_{S}$ affect the individual rates $R_{U}$ and $R_{W}$ as well as the weightings of $R_{U}$ and $R_{W}$ by the respective sizes of $U$ and $W$. First, consider $R_{U}$. The lines of $U$ are encoded independently with the respective moment-matching reduced MRF coding distributions. From the stationarity assumption, these moment-matching reduced MRFs are the same for each line, and therefore

$$
R_{U}=\frac{1}{|U|} H\left(X_{U} \| \tilde{X}_{U}\right)=\frac{1}{n_{L} N} H\left(X_{L} \| \tilde{X}_{L}\right)=\frac{1}{n_{L} N} H\left(X_{L}\right)+\frac{1}{n_{L} N} D\left(X_{L} \| \tilde{X}_{L}\right),
$$

where $L$ denotes a block of $n_{L}$ consecutive rows of the image, $X_{L}$ is the subset of the MRF on $L$, and $\tilde{X}_{L}$ is the same random variables with the moment-matching reduced MRF distribution. Next, by the Markov property and stationarity,

$$
R_{W}=\frac{1}{|W|} H\left(X_{W} \mid X_{U}\right)=\frac{1}{n_{S} N} H\left(X_{S} \mid X_{\partial S}\right)
$$

where $S$ denotes $n_{S}$ consecutive rows, $\partial S$ denotes the boundary of $S$, and $X_{S}$ and $X_{\partial S}$ are the respective subsets of random variables on $S$ and $\partial S$. Therefore, as a function of line and strip widths, the per-row rate $\biguplus^{3}$ is

$$
\bar{R}\left(n_{L}, n_{S}\right)=\frac{(k+1) n_{L}}{k n_{S}+(k+1) n_{L}} \frac{1}{n_{L}} H\left(X_{L} \| \tilde{X}_{L}\right)+\frac{k n_{S}}{k n_{S}+(k+1) n_{L}} \frac{1}{n_{S}} H\left(X_{S} \mid X_{\partial S}\right) .
$$

When $k$ is large, this is well approximated by

$$
\begin{aligned}
\bar{R}\left(n_{L}, n_{S}\right) & \approx \frac{n_{L}}{n_{L}+n_{S}} \frac{1}{n_{L}} H\left(X_{L} \| \tilde{X}_{L}\right)+\frac{n_{S}}{n_{L}+n_{S}} \frac{1}{n_{S}} H\left(X_{S} \mid X_{\partial S}\right) \\
& =\frac{n_{L}}{n_{L}+n_{S}} \frac{1}{n_{L}}\left(H\left(X_{L}\right)+D\left(X_{L} \| \tilde{X}_{L}\right)\right)+\frac{n_{S}}{n_{L}+n_{S}} \frac{1}{n_{S}} H\left(X_{S} \mid X_{\partial S}\right) .
\end{aligned}
$$

Intuitively, as the cutset line width $n_{L}$ increases, $R_{U}$ decreases because both $\frac{1}{n_{L}} H\left(X_{L}\right)$ and the divergence $\frac{1}{n_{L}} D\left(X_{L} \| \tilde{X}_{L}\right)$ would decrease. However, the fraction of sites $\frac{n_{L}}{n_{L}+n_{S}}$ encoded at the larger $R_{U}$ rate increases. Hence, there is a potential tradeoff between choosing $n_{L}$ to be large in order to reduce the cutset rate, and choosing $n_{L}$ to be small in order to reduce the fraction of sites in the cutset. Similarly, as $n_{S}$ increases, the fraction of pixels $\frac{n_{S}}{n_{L}+n_{S}}$ encoded at the lower rate increases, but one intuitively expects $\bar{R}_{W}=\frac{1}{n_{S}} H\left(X_{S} \mid X_{\partial S}\right)$ to increase. Again, a potential tradeoff.

On the other hand, since the overall rate is $R\left(n_{L}, n_{S}\right)=\frac{1}{|V|} H\left(X_{V}\right)+\frac{1}{|V|} D\left(X_{U} \| \tilde{X}_{U}\right)$, we see that the divergence term $\frac{1}{|V|} D\left(X_{U}|| \tilde{X}_{U}\right)$ is the redundancy of the code, and

\footnotetext{
${ }^{3}$ The overall rate is the per-row rate divided by the row width $N$. From now on, we mainly focus on per-row rate to simplify expressions, and use an overbar to indicate such.
} 
one can therefore focus on what makes it small. Letting $\Delta\left(n_{L}, n_{S}\right) \triangleq \frac{1}{|V|} D\left(X_{U}|| \tilde{X}_{U}\right)$ denote the redundancy of the code, we will show that the per-row redundancy has the form

$$
\begin{aligned}
\bar{\Delta}\left(n_{L}, n_{S}\right) & =\frac{(k+1) n_{L}}{k n_{S}+(k+1) n_{L}} \frac{1}{n_{L}} D\left(X_{L} \| \tilde{X}_{L}\right)+\frac{k n_{S}}{k n_{S}+(k+1) n_{L}} \frac{1}{n_{S}} I\left(X_{L_{i}} ; X_{L_{i-1}}\right) \\
& \approx \frac{n_{L}}{n_{L}+n_{S}} \frac{1}{n_{L}} D\left(X_{L} \| \tilde{X}_{L}\right)+\frac{n_{S}}{n_{L}+n_{S}} \frac{1}{n_{S}} I\left(X_{L_{i}} ; X_{L_{i-1}}\right)
\end{aligned}
$$

where $I\left(X_{L_{i}} ; X_{L_{i-1}}\right)$ is the mutual information between the random variables $X_{L_{i}}$ on a line and the random variables $X_{L_{i-1}}$ on the previous line. Note that in the above formula for redundancy, which is entirely due to the encoding of the lines, the first term, which we call the distribution redundancy is due to use of the reduce MRF coding distribution on each line and the second term, which we call the correlation redundancy is due the fact that lines are coded independently. Note also that while the redundancy is entirely due to encoding of the lines, the correlation redundancy depends on the strip width $n_{S}$. Moreover, since there is no correlation redundancy in the encoding of the first line, it is appropriate to think of $I\left(X_{L_{i}} ; X_{L_{i-1}}\right)$ as a penalty per strip. From this viewpoint, one would expect that increasing $n_{L}$ reduces the divergence per cutset pixel $\frac{1}{n_{L}} D\left(X_{L}|| \tilde{X}_{L}\right)$, but increases the fraction $\frac{n_{L}}{n_{L}+n_{S}}$ of the image included in the cutset. Hence, it is not clear what is the best value for $n_{L}$. Similarly, one would expect that information $I\left(X_{L_{i}} ; X_{L_{i-1}}\right)$ decreases in $n_{S}$, while the fraction of pixels $\frac{n_{S}}{n_{L}+n_{S}}$ increases in $n_{S}$. Therefore, it is likewise not clear what $n_{S}$ should be.

The results of this paper are to show the following results, most of which have been conjectured above. Under the stationarity assumption, the coding rate $R_{n_{S}}^{S}$ of a strip increases with $n_{S}$, the coding rate $R_{n_{L}}^{L}$ of a line decreases with $n_{L}$ when the momentmatching reduced MRF is used to encode the lines, and $R_{n_{S}}^{S}<R_{n_{L}}^{L}$ for all choices of $n_{S}$ and $n_{L}$. We also present a consistent estimation algorithm for the momentmatching parameter $\theta_{U}^{*}$. We show that the divergence $D\left(X_{U} \| \tilde{X}_{U}\right)$, equivalently the redundancy, can be decomposed into a correlation redundancy due to encoding the lines independently and a distribution redundancy due to approximating the lines as reduced MRFs, and present analysis of these two sources of redundancy. Numerical simulations with an Ising model illustrate the propositions.

In the rest of this paper, Section 2 provides background on MRFs and lossless coding and Section 3 provides an overview Reduced Cutset Coding in the current setting. Section 4 presents an estimation algorithm for $\theta_{U}^{*}$, Section 5 establishes the anticipated tradeoffs between cutset thickness and spacing, and finally, Section 6 discusses numerical simulations with an Ising model.

\section{Background}

We introduce notation for lossless coding of MRFs. 


\subsection{Graphs and Markov Random Fields}

A path in a graph $G=(V, E)$ is a sequence of nodes, each successive pair of nodes being joined by an edge in $E$. A graph is said to be connected if every pair of nodes $i, j \in V$ can be joined by some path, and disconnected otherwise. For any $U \subset V$, its boundary $\partial U$ is the set of nodes not in $U$ connected by an edge to a member of $U$. The subgraph $G_{U}=\left(U, E_{U}\right)$ induced by $U$ is the graph consisting of nodes and edges contained in $U$. Likewise, the subgraph $G_{V \backslash U}$ is obtained by removing $U$ and all edges incident to it from $G$. If $G_{V \backslash U}$ is disconnected, each maximal connected subset of $G_{V \backslash U}$ is called a component, and $G_{V \backslash U}$ is simply the collection of the (disjoint) subgraphs induced by the respective components. A subset $U \subset V$ is called a cutset if $G_{V \backslash U}$ consists of more than one component.

A family of MRFs is specified by an alphabet $\mathcal{X}$ and a vector statistic $t=\left(t_{i}, i \in\right.$ $\left.V ; t_{i, j},\{i, j\} \in E\right)$ defined on the site values at individual nodes and the endpoints of edges. $]^{4}$ That is, for a given image $\mathbf{x}=\left\{x_{i}: i \in V\right\}$, the function $t_{i j}: \mathcal{X} \times$ $\mathcal{X} \longrightarrow \mathbb{R}$ determines the contribution of the pair $\left(x_{i}, x_{j}\right)$ to the probability of $\mathbf{x}$, and similarly for $t_{i}: \mathcal{X} \longrightarrow \mathbb{R}$. We say that $X$ is an MRF based on $t$. The entire family of MRFs based on $t$ is generated by introducing an exponential parameter vector $\theta=\left(\theta_{i}, i \in V ; \theta_{i j},\{i, j\} \in E\right)$ where for each node $i$, and neighbor $j \in \partial i, \theta_{i}$ and $\theta_{i j}$ scale the sensitivity of the distribution $p(G ; \mathbf{x} ; \theta)$ to the functions $t_{i}$ and $t_{i j}$, respectively. Specifically, for an $\operatorname{MRF} X$ on $G$ based on $t$ with exponential parameter $\theta$, configuration $\mathbf{x}$ has probability $p(G ; \mathbf{x} ; \theta)$ given by

$$
p(G ; \mathbf{x} ; \theta)=\exp \{\langle\theta, t(\mathbf{x})\rangle-\Phi(\theta)\},
$$

where $\langle$,$\rangle denotes inner product, \Phi(\theta)$ is the log-partition function, and the arguments of $p(\cdot ; \cdot ; \cdot)$ indicate, respectively, the graph on which the MRF is defined, the configuration in question, and the exponential parameter on the graph. For a given exponential coordinate vector $\theta$, we let $\mu=\mu(\theta)$ denote the expected value of the statistic $t$ under the MRF induced by $\theta$, and we refer to $\mu$ as the moment of the MRF. The MRF distribution over all configurations is denoted $p(G ; X ; \theta)$, and the entropy of an MRF is denoted $H(G ; X ; \theta)$.

The conditional probability of a configuration $\mathbf{x}_{W}$ on subset $W \subset V$ given the values $\mathbf{x}_{U}$ on another subset $U \subset V$ is denoted $p\left(G ; \mathbf{x}_{W} \mid \mathbf{x}_{U} ; \theta\right)$. It is straightforward to check that $p\left(G ; \mathbf{x}_{W} \mid \mathbf{x}_{\partial W} ; \theta\right)=p\left(G ; \mathbf{x}_{W} \mid \mathbf{x}_{V \backslash W} ; \theta\right)$ for all $W, \mathbf{x}_{W}$, and $\mathbf{x}_{\partial W}$. This is the Markov Property. The conditional distributions of random subfield $X_{W}$ given a specific configuration $\mathbf{x}_{\partial W}$, or on the random subfield $X_{\partial W}$, are denoted $p\left(G ; X_{W} \mid \mathbf{x}_{\partial W} ; \theta\right)$ and $p\left(G ; X_{W} \mid X_{\partial W} ; \theta\right)$, respectively. Likewise, $H\left(G ; X_{W} \mid \mathbf{x}_{\partial W} ; \theta\right)$ and $H\left(G ; X_{W} \mid X_{\partial W} ; \theta\right)$ are the respective conditional entropies of $X_{W}$ given a specific configuration $\mathbf{x}_{\partial W}$ or the random subfield $X_{\partial W}$.

For subset $U$, the marginal probability distribution on $X_{U}$ is denoted $p\left(G ; X_{U} ; \theta\right)$, where $p\left(G ; \mathbf{x}_{U} ; \theta\right)$ denotes the marginal probability of configuration $\mathbf{x}_{U}$. The reduced $M R F$ distribution for $X_{U}$ on $G_{U}$ based on statistic $t_{U}$ with exponential parameter $\tilde{\theta}_{U}$ is denoted $p\left(G_{U} ; X_{U} ; \tilde{\theta}_{U}\right)$ and has the same form as in $(2)$, where $\Phi_{U}\left(\tilde{\theta}_{U}\right)$ denotes

\footnotetext{
${ }^{4}$ Properly, this is a pairwise MRF. Generalizations to other MRFs are straightforward.
} 


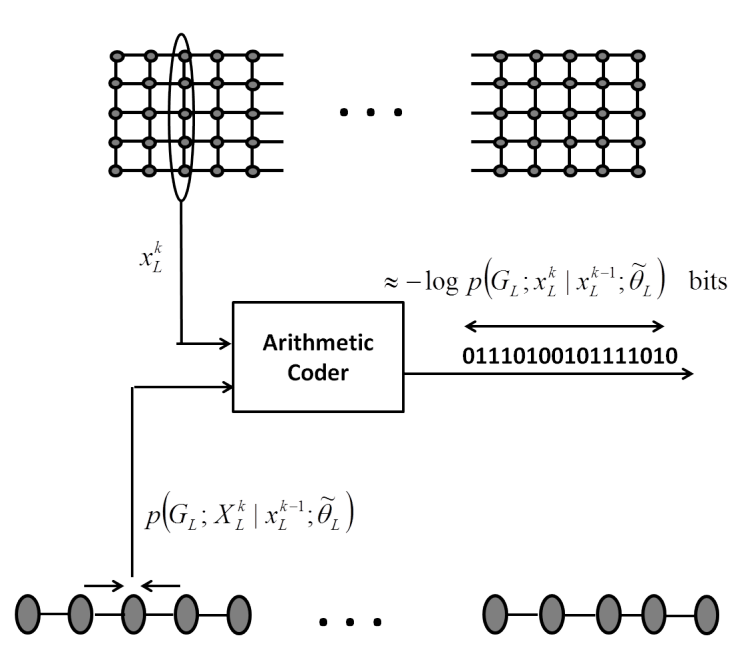

(a)

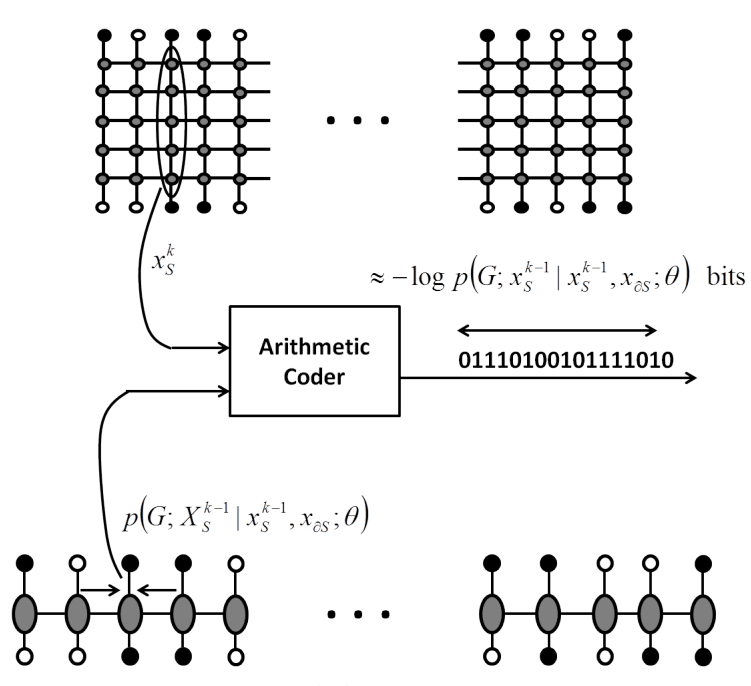

(b)

Figure 1: (a) AC encoding of a line $X_{L}$ with reduced MRF $P\left(G_{L} ; X_{L} ; \tilde{\theta}_{L}\right)$ coding distribution using Belief Propagation, and (b) AC encoding of a strip $X_{S}$ conditioned on its boundary $X_{\partial S}$ with conditional distribution $p\left(G ; X_{S} \mid X_{\partial S} ; \theta\right)$ using Belief Propagation.

the log-partition function for the reduced MRF. Similarly, $p\left(G_{U} ; \mathbf{x}_{U} ; \tilde{\theta}_{U}\right)$ denotes the probability of configurations $\mathbf{x}_{U}$ under the reduced MRF distribution. The statistic $t_{U}$ is inherited from the original statistic $t$. The marginal entropy of $X_{U}$ is denoted $H\left(G ; X_{U} ; \theta\right)$ while the entropy of a reduced $\operatorname{MRF} p\left(G_{U} ; X_{U} ; \tilde{\theta}_{U}\right)$ is denoted $H\left(G_{U} ; X_{U} ; \tilde{\theta}_{U}\right)$.

\subsection{Belief Propagation and Lossless Coding}

In general, ones uses Belief Propagation (BP) [14] to compute $p\left(G ; \mathbf{x}_{U} ; \theta\right)$ for a configuration $\mathbf{x}_{U}$. Since the inner product $\left\langle t_{U}\left(\mathbf{x}_{U}\right), \theta_{U}\right\rangle$ can be computed directly, BP is used to compute the log-partition function $\Phi(\theta)$, and more generally, to marginalize over $X_{V \backslash U}$. If $G$ has no cycles, then $p\left(G ; \mathbf{x}_{U} ; \theta\right)$ can be computed with complexity linear in the number of nodes in $V$. If $G$ has cycles, one can compute $p\left(G ; \mathbf{x}_{U} ; \theta\right)$ by grouping subsets of $V$ into supernodes such that the new graph is acyclic [14]. In this case, complexity is exponential in the size of the largest supernode. A graph is said to be tractable if either $G$ has no cycles or if $G$ can be clustered into an acyclic graph where the size of the largest supernode is moderate. Similarly, a subset $U$ is said to be tractable if $G_{U}$ is tractable, in which case $p\left(G_{U} ; \mathbf{x}_{U} ; \tilde{\theta}_{U}\right)$ can be computed for the reduced MRF on $G_{U}$. Also, for tractable subset $W, p\left(G ; \mathbf{x}_{W} \mid \mathbf{x}_{\partial W} ; \theta\right)$ can be computed for configurations $\mathbf{x}_{W}$ and $\mathbf{x}_{\partial W}$.

For the purposes of this paper it suffices to say that lossless compression with an optimal encoder involves computation of a coding distribution. For a tractable subset $U$, if configuration $\mathbf{x}_{U}$ is losslessly compressed with reduced MRF coding distribution $p\left(G_{U} ; X_{U} ; \tilde{\theta}_{U}\right)$, then the average number of bits produced is the cross entropy $H\left(G ; X_{U} ; \theta \| G_{U} ; X_{U} ; \tilde{\theta}_{U}\right)$ between the marginal distribution $p\left(G ; X_{U} ; \theta\right)$ and the re- 
duced MRF coding distribution $p\left(G_{U} ; X_{U} ; \tilde{\theta}_{U}\right)$ for $X_{U}$, defined as

$$
H\left(G ; X_{U} ; \theta \| G_{U} ; X_{U} ; \tilde{\theta}_{U}\right)=H\left(G ; X_{U} ; \theta\right)+D\left(p\left(G ; X_{U} ; \theta\right) \| p\left(G_{U} ; X_{U} ; \tilde{\theta}_{U}\right)\right)
$$

where $D\left(p\left(G ; X_{U} ; \theta\right) \| p\left(G_{U} ; X_{U} ; \tilde{\theta}_{U}\right)\right)$ is the divergence from $p\left(G ; X_{U} ; \theta\right)$ to $p\left(G_{U} ; X_{U} ; \tilde{\theta}_{U}\right)$ and is the redundancy in the code [4].

We showed in [10] that the above divergence is minimized at $\theta_{U}^{*}$, the exponential parameter on $G_{U}$ such that the corresponding moment $\mu_{U}^{*}$ is equal to the moment subvector $\mu_{U}$ under the original MRF $p(G ; X ; \theta)$. The distribution of the reduced MRF $p\left(G_{U} ; X_{U} ; \theta_{U}^{*}\right)$ is called the moment-matching reduced MRF distribution for $X_{U}$, denoted $\tilde{X}_{U}$. When the moment-matching reduced MRF $p\left(G_{U} ; X_{U} ; \theta_{U}^{*}\right)$ is used as the coding distribution to encode $X_{U}$, the cross entropy is in fact the entropy $H\left(G_{U} ; X_{U} ; \theta_{U}^{*}\right)$ of the moment-matching reduced MRF [10].

For a tractable subset $W$, if configuration $\mathbf{x}_{W}$ is encoded conditioned on $\mathbf{x}_{\partial W}$ using coding distribution $p\left(G ; X_{W} \mid \mathbf{x}_{\partial W} ; \theta\right)$, then the average number of bits produced is $H\left(G ; X_{W} \mid X_{\partial W} ; \theta\right)$. Therefore, encoding $\mathbf{x}_{W}$ conditioned on $\mathbf{x}_{\partial W}$ is optimal, i.e., there is no redundancy.

In [10], Arithmetic Coding (AC) was proposed as the optimal encoder. Figure 1 illustrates the encoding of a line and a strip. The mathematical details of using $\mathrm{AC}$ in the encoding of an MRF are given in [8], [10], and [11], specifically in Chapter VI of $[1]$.

\section{Reduced Cutset Coding}

In general, since the cutset $U$ consists of disjoint lines, the entropy of the momentmatching reduced MRF on $G_{U}$ is actually the sum $\sum_{L_{i}} H\left(G_{L_{i}} ; X_{L_{i}} ; \theta_{L_{i}}^{*}\right)$ of the entropies of the reduced MRFs on the individual lines. Similarly, the conditional entropy of $X_{W}$ given $X_{\partial W}$ is the sum $\sum_{S_{i}} H\left(G ; X_{S_{i}} \mid X_{\partial S_{i}} ; \theta\right)$ of the conditional entropies of the individuals strips given their respective boundaries.

In the present paper, we simplify this by considering vertically homogeneous parameters for the MRF, i.e., the components of the statistic $t$ and the exponential parameter $\theta$ do not vary vertically within the image. Furthermore, focusing only on the middle $M^{\prime}=\left(k^{\prime}+1\right) n_{L}+k^{\prime} n_{S} \approx M / 2$ rows of $V$, therefore excluding boundary effects, the image will be roughly stationary in the vertical direction. We let $B_{n}$ be an $n \times N$ rectangular subset of sites.

The random field $X_{B_{n_{L}}}$ on a line is encoded with reduced MRF coding distribution $p\left(G_{n_{L}} ; X_{B_{n_{L}}} ; \theta_{B_{n_{L}}}^{*}\right)$. Normalizing by the number of pixels, the per-row rate for encoding a line is then

$$
\begin{aligned}
\bar{R}_{n_{L}}^{L} & =\frac{1}{n_{L}} H\left(G ; X_{B_{n_{L}}} ; \theta \| G_{n_{L}} ; X_{B_{n_{L}}} ; \theta_{B_{n_{L}}}^{*}\right) \\
& =\frac{1}{n_{L}} H\left(G_{B_{n_{L}}} ; X_{B_{n_{L}}} ; \theta_{B_{n_{L}}}^{*}\right) .
\end{aligned}
$$

The random field $X_{B_{n_{S}}}$ on a strip is encoded conditioned on $X_{\partial B_{n_{S}}}$ with coding 
distribution $p\left(G ; X_{B_{n_{S}}} \mid X_{\partial B_{n_{S}}} ; \theta\right)$. The per-row rate for encoding a strip is then

$$
\bar{R}_{n_{S}}^{S}=\frac{1}{n_{S}} H\left(G ; X_{B_{n_{S}}} \mid X_{\partial B_{n_{S}}} ; \theta\right)
$$

We let $\bar{R}_{n_{S}, n_{L}}$ denote the total per-row rate of RCC with cutset parameters $n_{S}$ and $n_{L}$, given by

$$
\bar{R}\left(n_{S}, n_{L}\right)=\frac{(k+1) n_{L}}{(k+1) n_{L}+k n_{S}} \bar{R}_{n_{L}}^{L}+\frac{k n_{S}}{(k+1) n_{L}+k n_{S}} \bar{R}_{n_{S}}^{S} .
$$

Assuming further that $M^{\prime}$ is very large relative to $n_{L}$ and $n_{S}$, so that $k$ is very large, this rate is well-approximated by

$$
\bar{R}\left(n_{S}, n_{L}\right) \approx \frac{n_{L}}{n_{L}+n_{S}} \bar{R}_{n_{L}}^{L}+\frac{n_{S}}{n_{L}+n_{S}} \bar{R}_{n_{S}}^{S}
$$

We now see that the performance of RCC with cutset parameters $n_{S}$ and $n_{L}$ is characterized by the rates $\bar{R}_{n_{L}}^{L}$ and $\bar{R}_{n_{S}}^{S}$, and the fractions $\frac{n_{L}}{n_{L}+n_{S}}$ and $\frac{n_{S}}{n_{L}+n_{S}}$.

\section{Moment-matching $\theta_{U}^{*}$}

Recall from the previous section that the cross-entropy $H\left(p\left(G ; X_{U} ; \theta\right) \| p\left(G_{U} ; X_{U} ; \tilde{\theta}_{U}\right)\right.$ between the marginal distribution $p\left(G ; X_{U} ; \theta\right)$ of subset $X_{U}$ within an MRF on $G$ with statistic $t$ and a reduced MRF $p\left(G_{U} ; X_{U} ; \tilde{\theta}_{U}\right)$ on $G_{U}$ with statistic $t_{U}$ is minimized by the parameter $\theta_{U}^{*}$ such that the expected value $\mathbb{E}_{\theta_{U}^{*}}\left[t_{U}\left(X_{U}\right)\right]$ of the statistic $t_{U}$ in the reduced MRF equals the expected value $\left(\mathbb{E}_{\theta}[t(X)]\right)_{U}$ of the statistic $t$ under the original MRF on the subset $U$, referred to as the moment-matching parameter. We will estimate $\theta_{U}^{*}$ from $n$ observations $\mathbf{x}_{U}^{(1)}, \ldots, \mathbf{x}_{U}^{(n)}$ on $U$, by seeking an $\hat{\theta}_{U}^{n}$ that minimizes an empirical version of the cross entropy, at least approximately. First, some background.

We let $\Theta=\{\theta\}$ denote the set of parameter vectors for MRFs on $G$ based on the statistic $t$. We restrict attention to the case where $\Theta$ is the subset of $\mathbb{R}^{|V|+|E|}$ of $\theta^{\prime}$ s with positive components. In this case, due to the openness of $\Theta$, the family of MRFs based on $t$ is said to be regular [14]. For parameter $\theta \in \Theta$, the function

$$
\begin{aligned}
\Lambda(\theta) & \triangleq \mathbb{E}_{\theta}[t(X)] \\
& \triangleq \mu
\end{aligned}
$$

maps $\theta$ to $\mu$, the expected value of $t$ under the MRF induced by $\theta$, referred to as the moment of the MRF. The set $\mathcal{M}=\{\mu=\Lambda(\theta): \theta \in \Theta\}$ is the set of achievable moments for MRFs on $G$ based on $t$. We assume that the statistic $t$ is minimal in that the components of $t$ are affinely independent, meaning that the components of $t(\mathbf{x})$ do not sum to a constant for all configurations $\mathbf{x}$. In this case, the function $\Lambda(\cdot)$ is one-to-one [14]. Then, for $\mu \in \mathcal{M}$, the inverse function

$$
\Lambda^{-1}(\mu)=\theta
$$


is well-defined. Moreover, $\mu$ is a dual parameter to $\theta$, in that the $\operatorname{MRF} p(G ; X ; \theta)$ can alternatively be expressed as $p(G ; X ; \mu)$. For the MRF induced by parameter $\theta$, the subvector of moments on the set $U$ is given by

$$
\Lambda_{U}(\theta)=\mu_{U}
$$

which can be seen as the restriction of $\Lambda(\cdot)$ to the set $U$.

For reduced MRFs on $G_{U}$ based on statistic $t_{U}, \tilde{\Theta}_{U}$ denotes the associated set of exponential parameters. Now, consider the function

$$
\tilde{\Lambda}_{U}\left(\tilde{\theta}_{U}\right)=\tilde{\mu}_{U}
$$

which maps a parameter $\tilde{\theta}_{U} \in \tilde{\Theta}_{U}$ to the corresponding moment $\tilde{\mu}_{U}$ for the reduced MRF $p\left(G_{U} ; X_{U} ; \tilde{\theta}_{U}\right)$ on $G_{U}$. Likewise, $\tilde{\mathcal{M}}_{U}=\left\{\tilde{\mu}_{U}=\tilde{\Lambda}_{U}\left(\tilde{\mu}_{U}\right): \tilde{\theta}_{U} \in \tilde{\Theta}_{U}\right\}$ denotes the set of achievable moments for reduced MRFs on $G_{U}$. Since we have assumed that the statistic $t$ for the original family of MRFs on $G$ is minimal, the statistic $t_{U}$ for the family of reduced MRFs on $G_{U}$ is also minimal, and the inverse map $\tilde{\Lambda}_{U}^{-1}\left(\tilde{\mu}_{U}\right)=\tilde{\theta}_{U}$ is well-defined. Again, a reduced $\operatorname{MRF} p\left(G_{U} ; X_{U} ; \tilde{\theta}_{U}\right)$ can also be parameterized as $p\left(G_{U} ; X_{U} ; \tilde{\mu}_{U}\right)$.

Given a parameter $\theta$ for an $\operatorname{MRF} p(G ; X ; \theta)$, a subset $U$, and a sequence of observations $\mathbf{x}_{U}^{(1)}, \ldots, \mathbf{x}_{U}^{(n)}$ on $U$, we define the empirical moment of $p\left(G ; X_{U} ; \theta\right)$ as

$$
\hat{\mu}_{U}^{n} \triangleq \frac{1}{n} \sum_{i=1}^{n} t_{U}\left(\mathbf{x}_{U}^{(i)}\right)
$$

While $\mu_{U}=\Lambda_{U}(\theta)$ is always contained in $\tilde{\mathcal{M}}_{U}$, it is not necessarily the case that the empirical moment $\hat{\mu}_{U}^{n}$ is contained in $\tilde{\mathcal{M}}_{U}$. However, even if $\hat{\mu}_{U}^{n}$ is not in $\tilde{\mathcal{M}}_{U}$, $\hat{\mu}_{U}^{n}$ is still a limit point of $\left.\tilde{\mathcal{M}}_{U} \sqrt{14}\right]$, meaning that for every $\epsilon>0$, there is an $\epsilon$-ball containing $\hat{\mu}_{U}^{n}$ that contains infinitely many points of $\tilde{\mathcal{M}}_{U}$. Moreover, as stated in the following proposition, as the number of observations $n$ approaches $\infty$, not only is $\hat{\mu}_{U}^{n}$ in $\tilde{\mathcal{M}}_{U}$, but $\hat{\mu}_{U}^{n}$ converges to $\mu_{U}$.

Proposition 4.1 The empirical moment $\hat{\mu}_{U}^{n}$ converges in probability to $\mu_{U}$, i.e., for any $\epsilon>0$,

$$
\operatorname{Pr}\left(\left|\hat{\mu}_{U}^{n}-\mu_{U}\right| \leq \epsilon\right) \rightarrow 1 \text {, as } n \rightarrow \infty
$$

Proof To prove the proposition, one should recall that on a finite graph $G$, there does not exist a phase transition [5], and therefore, there is a unique MRF on $G$ for the specified statistic $t$ and exponential parameter $\theta$. It follows that the sequence $\mathbf{x}_{U}^{(1)}, \ldots, \mathbf{x}_{U}^{(n)}, \ldots$ is not only stationary but also ergodic, from which the proposition follows $[6]$. This completes the proof. 


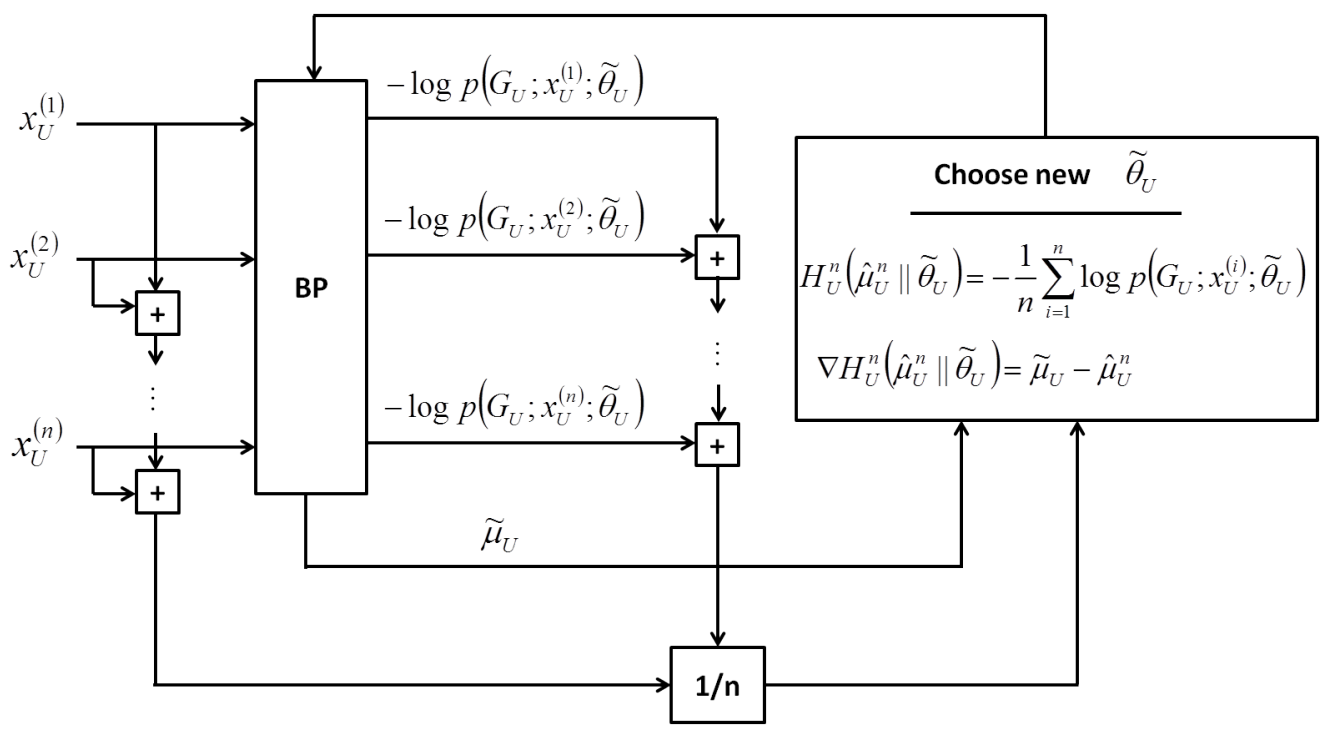

Figure 2: Block diagram for finding the moment-matching parameter $\theta_{U}^{*}$ for encoding $X_{U}$.

We now discuss the empirical version of cross entropy that we will minimize as a surrogate for cross entropy. From a sequence of observations $\mathbf{x}_{U}^{(1)}, \ldots, \mathbf{x}_{U}^{(n)}$, we define the empirical cross entropy

$$
\begin{aligned}
H_{U}^{n}\left(\hat{\mu}_{U}^{n} \| \tilde{\theta}_{U}\right) & \triangleq-\frac{1}{n} \sum_{i=1}^{n} \log p\left(G_{U} ; \mathbf{x}_{U}^{(i)} ; \tilde{\theta}_{U}\right) \\
& =-\sum_{\mathbf{x}_{U}} f\left(\mathbf{x}_{U}: \mathbf{x}_{U}^{(1)}, \ldots, \mathbf{x}_{U}^{(n)}\right) \log p\left(G_{U} ; \mathbf{x}_{U} ; \tilde{\theta}_{U}\right)
\end{aligned}
$$

between the empirical distribution $f\left(\mathbf{x}_{U}: \mathbf{x}_{U}^{(1)}, \ldots, \mathbf{x}_{U}^{(n)}\right)$ generated by $\mathbf{x}_{U}^{(1)}, \ldots, \mathbf{x}_{U}^{(n)}$ and the reduced MRF $p\left(G_{U} ; X_{U} ; \tilde{\theta}_{U}\right)$ induced by a candidate parameter $\tilde{\theta}_{U}$. That it makes sense to consider the empirical cross entropy to be a function of the empirical moment $\hat{\mu}_{U}^{n}$ is due to the proposition presented later. If $U$ is a tractable subset, then the probabilities in the summation can be efficiently computed.

Now, our estimate for the moment-matching parameter $\theta_{U}^{*}$ will be the $\hat{\theta}_{U}^{n}$ that minimizes this empirical cross entropy, at least approximately. It is well-known that $\Phi_{U}\left(\tilde{\theta}_{U}\right)$ is convex in $\tilde{\theta}_{U}$, and, as follows from the following theorem, so is the empirical cross-entropy $H_{U}^{n}\left(\hat{\mu}_{U}^{n} \| \tilde{\theta}_{U}\right)$. If, as we have assumed, the components of $t_{U}$ are affinely independent, then $\Phi_{U}\left(\tilde{\theta}_{U}\right)$ and hence $H_{U}^{n}\left(\hat{\mu}_{U}^{n} \| \tilde{\theta}_{U}\right)$ is strictly convex. Therefore, either $H_{U}^{n}\left(\hat{\mu}_{U}^{n} \| \tilde{\theta}_{U}\right)$ has a unique minimum at a $\tilde{\theta}_{U}$ at which the gradient of $H_{U}^{n}\left(\hat{\mu}_{U}^{n} \| \tilde{\theta}_{U}\right)$ is zero, or since $\tilde{\Theta}_{U}$ is open, $H_{U}^{n}\left(\hat{\mu}_{U}^{n} \| \tilde{\theta}_{U}\right)$ does not have a minimum but approaches an infimum at a limit point of $\tilde{\Theta}_{U}$. Moreover, from the following theorem and the fact that for any $\hat{\mu}_{U}^{n}$ there exists $\tilde{\theta}_{U}$ such that $\tilde{\Lambda}_{U}\left(\tilde{\theta}_{U}\right)$ is arbitrarily close to $\hat{\mu}_{U}^{n}$, we can find $\tilde{\theta}_{U}$ such that the gradient is arbitrarily small and such $\tilde{\theta}_{U}$ must come arbitrarily close to attaining the infimum of $H_{U}^{n}\left(\hat{\mu}_{U}^{n} \| \tilde{\theta}_{U}\right)$. In either case, our "moment-matching" estimate $\hat{\theta}_{U}^{n}$ will be a $\tilde{\theta}_{U}$ that induces a very small gradient. 


\section{Proposition 4.2}

$$
\begin{aligned}
H_{U}^{n}\left(\hat{\mu}_{U}^{n}|| \tilde{\theta}_{U}\right) & =\Phi_{U}\left(\tilde{\theta}_{U}\right)-\left\langle\hat{\mu}_{U}^{n}, \tilde{\theta}_{U}\right\rangle \\
\nabla H_{U}^{n}\left(\hat{\mu}_{U}^{n}|| \tilde{\theta}_{U}\right) & =\tilde{\mu}_{U}-\hat{\mu}_{U}^{n} \\
& =\tilde{\Lambda}_{U}\left(\tilde{\theta}_{U}\right)-\hat{\mu}_{U}^{n},
\end{aligned}
$$

where the gradient is with respect to $\tilde{\theta}_{U}$.

Proof Using relation (2) for the reduced MRF on $G_{U}$ with parameter $\tilde{\theta}_{U}$, we get

$$
\begin{aligned}
H_{U}^{n}\left(\hat{\mu}_{U}^{n} \| \tilde{\theta}_{U}\right) & =\frac{1}{n} \sum_{i=1}^{n}\left[\Phi_{U}\left(\tilde{\theta}_{U}\right)-\left\langle t\left(\mathbf{x}_{U}^{(i)}\right), \tilde{\theta}_{U}\right\rangle\right] \\
& =\Phi_{U}\left(\tilde{\theta}_{U}\right)-\left\langle\sum_{i=1}^{n} t\left(\mathbf{x}_{U}^{(i)}\right), \tilde{\theta}_{U}\right\rangle \\
& =\Phi_{U}\left(\tilde{\theta}_{U}\right)-\left\langle\hat{\mu}_{U}^{n}, \tilde{\theta}_{U}\right\rangle
\end{aligned}
$$

It is well-known that $\nabla \Phi_{U}\left(\tilde{\theta}_{U}\right)=\tilde{\mu}_{U} \mid 14$. Then, taking the gradient of $H_{U}^{n}\left(\hat{\mu}_{U}^{n} \| \tilde{\theta}_{U}\right)$ yields

$$
\begin{aligned}
\nabla H_{U}^{n}\left(\hat{\mu}_{U}^{n}|| \tilde{\theta}_{U}\right) & =\nabla \frac{1}{n} \sum_{i=1}^{n}\left[\Phi_{U}\left(\tilde{\theta}_{U}\right)-\left\langle t_{U}\left(\mathbf{x}_{U}^{(i)}\right), \tilde{\theta}_{U}\right\rangle\right] \\
& =\nabla \Phi_{U}\left(\tilde{\theta}_{U}\right)-\nabla \frac{1}{n} \sum_{i=1}^{n}\left\langle t_{U}\left(\mathbf{x}_{U}^{(i)}\right), \tilde{\theta}_{U}\right\rangle \\
& =\nabla \Phi_{U}\left(\tilde{\theta}_{U}\right)-\nabla\left\langle\frac{1}{n} \sum_{i=1}^{n} t_{U}\left(\mathbf{x}_{U}^{(i)}\right), \tilde{\theta}_{U}\right\rangle \\
& =\tilde{\mu}_{U}-\frac{1}{n} \sum_{i=1}^{n} t_{U}\left(\mathbf{x}_{U}^{(i)}\right) \\
& =\tilde{\mu}_{U}-\hat{\mu}_{U}^{n} .
\end{aligned}
$$

This completes the proof.

We now describe how a gradient descent algorithm can be used to find an estimate $\hat{\theta}_{U}^{n}$ of $\theta_{U}^{*}$ at which the gradient of $H_{U}^{n}\left(\hat{\mu}_{U}^{n} \| \tilde{\theta}_{U}\right)$ is arbitrarily small. From the sequence $\mathbf{x}_{U}^{(1)}, \ldots, \mathbf{x}_{U}^{(n)}$, we first compute the empirical moment $\hat{\mu}_{U}^{n}=\frac{1}{n} \sum_{i=1}^{n} t_{U}\left(\mathbf{x}_{U}^{(i)}\right)$. Then, given a candidate parameter $\tilde{\theta}_{U}$, use Belief Propagation to compute the negative $\log$-likelihood $-\log p\left(G_{U} ; \mathbf{x}_{U}^{(i)} ; \tilde{\theta}_{U}\right)$ of the configuration $\mathbf{x}^{(i)}$ under the reduced MRF $p\left(G_{U} ; X_{U} ; \tilde{\theta}_{U}\right)$, for each $i=1, \ldots, n$. Additionally, we compute the moment $\tilde{\mu}_{U}$ of the reduced MRF induced by the candidate parameter $\tilde{\theta}_{U}$, which like the probabilities, can be computed due to tractability of $U$. We then compute the objective function 
$H_{U}^{n}\left(\hat{\mu}_{U}^{n}|| \tilde{\theta}_{U}\right)=-\frac{1}{n} \sum_{i=1}^{n} \log p\left(G_{U} ; \mathbf{x}_{U}^{(i)} ; \tilde{\theta}_{U}\right)$ and the gradient $\nabla H_{U}^{n}\left(\hat{\mu}_{U}^{n} \| \tilde{\theta}_{U}\right)=\tilde{\mu}_{U}-\hat{\mu}_{U}^{n}$. Finally, given a tolerance $\epsilon_{\mu}$, if $\left\|\nabla H_{U}^{n}\left(\hat{\mu}_{U}^{n} \| \tilde{\theta}_{U}\right)\right\|<\epsilon_{\mu}$, the algorithm terminates and we set $\hat{\theta}_{U}^{n}=\tilde{\theta}_{U}$ which corresponds to the estimated moment $\hat{\hat{\mu}}_{U}^{n}=\tilde{\Lambda}_{U}\left(\hat{\theta}_{U}^{n}\right)$ at which the algorithm is terminated. Note that by Proposition 4.2, the estimated moment $\hat{\hat{\mu}}_{U}^{n}$ is within $\epsilon_{\mu}$ of $\hat{\mu}_{U}^{n}$. If $\left\|\nabla H_{U}^{n}\left(\hat{\mu}_{U}^{n} \| \tilde{\theta}_{U}\right)\right\| \geq \epsilon_{\mu}$, we determine a new candidate parameter $\tilde{\theta}_{U}$ using a standard gradient descent method $[3]$ and repeat the above steps. This is illustrated in Figure 2.

Proposition 4.3 The estimate $\hat{\theta}_{U}^{n}$ is consistent, i.e., for any $\epsilon>0$,

$$
\operatorname{Pr}\left(\left|\hat{\theta}_{U}^{n}-\theta_{U}^{*}\right| \leq \epsilon\right) \rightarrow 1 \text {, as } n \rightarrow \infty .
$$

Proof Let $B\left(\theta_{U}^{*}, \epsilon_{\theta}\right)$ be the $\epsilon_{\theta}$-ball centered at $\theta_{U}^{*}$. Assume without loss of generality that $B\left(\theta_{U}^{*}, \epsilon_{\theta}\right) \subset \tilde{\Theta}_{U}$. Then, let $\epsilon_{\mu}$ be the largest tolerance around $\mu_{U}$ such that the $\epsilon_{\mu}$-ball $B\left(\mu_{U}, \epsilon_{\mu}\right)$ centered at $\mu_{U}$ is contained in $\tilde{\Lambda}_{U}\left(B\left(\theta_{U}^{*}, \epsilon_{\theta}\right)\right)$. It follows that

$$
\begin{aligned}
\operatorname{Pr}\left(\left|\hat{\theta}_{U}^{n}-\theta_{U}^{*}\right| \leq \epsilon_{\theta}\right) & =\operatorname{Pr}\left(\hat{\hat{\mu}}_{U}^{n} \in \tilde{\Lambda}_{U}\left(B\left(\theta_{U}^{*}, \epsilon_{\theta}\right)\right)\right) \\
& \geq \operatorname{Pr}\left(\hat{\hat{\mu}}_{U}^{n} \in B\left(\mu_{U}, \epsilon_{\mu}\right)\right) \\
& =\operatorname{Pr}\left(\left|\hat{\hat{\mu}}_{U}^{n}-\mu_{U}\right| \leq \epsilon_{\mu}\right) .
\end{aligned}
$$

Now let $\epsilon_{\mu}^{\prime}=\epsilon_{\mu} / 2$ be the tolerance on $\left\|\nabla H_{U}^{n}\left(\hat{\mu}_{U}^{n} \| \tilde{\theta}_{U}\right)\right\|$ in the gradient descent algorithm. This means that $\left|\hat{\hat{\mu}}_{U}^{n}-\hat{\mu}_{U}^{n}\right| \leq \epsilon_{\mu}^{\prime}$, which in turn implies that

$$
\operatorname{Pr}\left(\left|\hat{\hat{\mu}}_{U}^{n}-\mu_{U}\right| \leq \epsilon_{\mu}\right)=\operatorname{Pr}\left(\left|\hat{\mu}_{U}^{n}-\mu_{U}\right| \leq \epsilon_{\mu}^{\prime}\right) .
$$

Using Proposition 4.1, we can now say that for an arbitrary tolerance $\delta>0$, there exists $N$ such that if the number of observations $n$ is greater than or equal to $N$, then

$$
\begin{aligned}
\operatorname{Pr}\left(\left|\hat{\theta}_{U}^{n}-\theta_{U}^{*}\right| \leq \epsilon_{\theta}\right) & \geq \operatorname{Pr}\left(\left|\hat{\mu}_{U}^{n}-\mu_{U}\right| \leq \epsilon_{\mu}^{\prime}\right) \\
& \geq 1-\delta .
\end{aligned}
$$

This completes the proof.

\section{$5 \quad$ Tradeoffs between Lines and Strips}

The following proposition shows that, as intuited earlier, strip rate increases with strip width.

\section{Proposition 5.1}

$$
\bar{R}_{n+1}^{S}>\bar{R}_{n}^{S}
$$


Lemma 5.2 Let $r_{1}$ denote the first row of rectangular region $B_{n}$ of sites of height $n$.

Then,

$$
H\left(G ; X_{r_{1}} \mid X_{\partial B_{n}} ; \theta\right)<H\left(G ; X_{r_{1}} \mid X_{\partial B_{n+1}} ; \theta\right)
$$

Proof Note $B_{n+1}$ consists of $B_{n}$ and an additional row $r_{n+1}$, which is part of the boundary of $B_{n}$. By the Markov property, $H\left(G ; X_{r_{1}} \mid X_{\partial B_{n}} ; \theta\right)=H\left(G ; X_{r_{1}} \mid X_{\partial B_{n+1}}, X_{r_{n+1}} ; \theta\right)$. That is, conditioning on $\partial B_{n+1}$ and $r_{n+1}$ is the same as conditioning on $\partial B_{n}$. Finally, $H\left(G ; X_{r_{1}} \mid X_{\partial B_{n+1}}, X_{r_{n+1}} ; \theta\right)<H\left(G ; X_{r_{1}} \mid X_{\partial B_{n+1}} ; \theta\right)$ as the left side has more conditioning. In summary

$$
\begin{aligned}
H\left(G ; X_{r_{1}} \mid X_{\partial B_{n}} ; \theta\right) & =H\left(G ; X_{r_{1}} \mid X_{\partial B_{n+1}}, X_{r_{n+1}} ; \theta\right) \\
& <H\left(G ; X_{r_{1}} \mid X_{\partial B_{n+1}} ; \theta\right) .
\end{aligned}
$$

This completes the proof of Lemma 5.2

We continue with the proof of Proposition 5.1.

Proof By direct calculation we have for a strip of height $n+1$ that

$$
\begin{aligned}
\bar{R}_{n+1}^{S} & =\frac{1}{(n+1)} H\left(G ; X_{B_{n+1}} \mid X_{\partial B_{n+1}} ; \theta\right) \\
& =\frac{1}{(n+1)} H\left(G ; X_{B_{n}} \mid X_{\partial B_{n}} ; \theta\right)+\frac{1}{(n+1)} H\left(G ; X_{r_{1}} \mid X_{\partial B_{n+1}} ; \theta\right),
\end{aligned}
$$

and for a strip of height $n$,

$$
\begin{aligned}
\bar{R}_{n}^{S} & =\frac{1}{n} H\left(G ; X_{B_{n}} \mid X_{\partial B_{n}} ; \theta\right) \\
& =\frac{n+1}{n} \frac{1}{(n+1)} H\left(G ; X_{B_{n}} \mid X_{\partial B_{n}} ; \theta\right) \\
& =\frac{1}{(n+1)} H\left(G ; X_{B_{n}} \mid X_{\partial B_{n}} ; \theta\right)+\frac{1}{n(n+1)} H\left(G ; X_{B_{n}} \mid X_{\partial B_{n}} ; \theta\right) \\
& =\frac{1}{(n+1)} H\left(G ; X_{B_{n}} \mid X_{\partial B_{n}} ; \theta\right)+\frac{1}{n} \sum_{i=1}^{n} \frac{1}{(n+1)} H\left(G ; X_{r_{i}} \mid X_{\partial B_{n-i+1}} ; \theta\right) \\
& <\frac{1}{(n+1)} H\left(G ; X_{B_{n}} \mid X_{\partial B_{n}} ; \theta\right)+\frac{1}{(n+1)} H\left(G ; X_{r_{1}} \mid X_{\partial B_{n+1}} ; \theta\right) \\
& =\bar{R}_{n+1}^{S}
\end{aligned}
$$

by (7) and Lemma 5.2. This completes the proof.

Likewise, the next proposition shows that, as supposed earlier, line rate decreases with line width. 


\section{Proposition 5.3}

$$
\bar{R}_{n+1}^{L}<\bar{R}_{n}^{L}
$$

Proof First we note that reducing $X_{B_{n+1}}$ to $\tilde{X}_{B_{n+1}}$ by matching moments and further reducing the $X_{B_{n}}$ marginal of $\tilde{X}_{B_{n+1}}$ to $\tilde{X}_{B_{n}}$ by matching moments results in the same reduced MRF on $G_{B_{n}}$ as would reducing the original $X_{B_{n}}$ to $\tilde{X}_{B_{n}}$ by matching moments. Let $\theta_{n}^{*}$ be the moment matching parameter for $\tilde{X}_{B_{n}}$.

$$
\begin{aligned}
\bar{R}_{n+1}^{l} & =\frac{1}{n+1} H\left(G_{B_{n+1}} ; X_{B_{n+1}} ; \theta_{n+1}^{*}\right) \\
& =\frac{1}{n+1}\left[H\left(G_{B_{n+1}} ; X_{B_{n}} ; \theta_{n+1}^{*}\right)+H\left(G_{B_{n+1}} ; X_{r_{n+1}} \mid X_{B_{n}} ; \theta_{n+1}^{*}\right)\right] \\
& <\frac{1}{n+1}\left[H\left(G_{B_{n+1}} ; X_{B_{n}} ; \theta_{n+1}^{*}\right)+\frac{1}{n} H\left(G_{B_{n+1}} ; X_{B_{n}} ; \theta_{n+1}^{*}\right)\right] \\
& =\frac{1}{n} H\left(G_{B_{n+1}} ; X_{B_{n}} ; \theta_{n+1}^{*}\right) \\
& <\frac{1}{n} H\left(G_{B_{n}} ; X_{B_{n}} ; \theta_{n}^{*}\right) \\
& =\bar{R}_{n}^{L},
\end{aligned}
$$

where the second inequality is from the maximum entropy property of MRFs. This completes the proof

Proposition 5.4 For all strip widths $n_{S}$ and line widths $n_{L}$,

$$
\bar{R}_{n_{L}}^{L}>\bar{R}_{n_{S}}^{S}
$$

Proof We prove the proposition by cases: $n_{S}=n_{L}, n_{S}>n_{L}$, and $n_{S}<n_{L}$.

First assume $n_{S}=n_{L}=n$. Then,

$$
\begin{aligned}
\bar{R}_{n_{S}}^{S} & =\frac{1}{n} H\left(G ; X_{B_{n}} \mid X_{\partial B_{n}} ; \theta\right) \\
& \leq \frac{1}{n} H\left(G ; X_{B_{n}} ; \theta\right) \\
& <\frac{1}{n} H\left(G_{B_{n}} ; X_{B_{n}} ; \theta_{n}^{*}\right) \\
& =\bar{R}_{n_{L}}^{L}
\end{aligned}
$$


where (8) follows from the maximum entropy property of MRFs. Next, assume $n_{S}>$ $n_{L}$. Then,

$$
\begin{aligned}
\bar{R}_{n_{S}}^{S} & =\frac{1}{n_{S}} H\left(G ; X_{B_{n}} \mid X_{\partial B_{n}} ; \theta\right) \\
& \leq \frac{1}{n_{S}} H\left(G ; X_{B_{n}} ; \theta\right) \\
& <\frac{1}{n_{S}} H\left(G_{B_{n}} ; X_{B_{n_{S}}} ; \theta_{n_{S}}^{*}\right) \\
& =\bar{R}_{n_{S}}^{L} \\
& <\bar{R}_{n_{L}}^{L},
\end{aligned}
$$

where (9) follows from the maximum entropy property of MRFs. Finally, assume $n_{S}<n_{L}$. Then,

$$
\begin{aligned}
\bar{R}_{n_{S}}^{S} & <\bar{R}_{n_{L}}^{S} \\
& =\frac{1}{n_{L}} H\left(G ; X_{B_{n_{L}}} \mid X_{\partial B_{n_{L}}} ; \theta\right) \\
& \leq \frac{1}{n_{L}} H\left(G ; X_{B_{n_{L}}} ; \theta\right) \\
& <\frac{1}{n_{L}} H\left(G_{B_{n_{L}}} ; X_{B_{n_{L}}} ; \theta_{n_{L}}^{*}\right) \\
& =\bar{R}_{n_{L}}^{L},
\end{aligned}
$$

where 10 follows from the maximum entropy property of MRFs. This completes the proof.

Together these three propositions indicate that $\bar{R}_{n_{L}}^{L}$ and $\bar{R}_{n_{S}}^{S}$ always behave as in Figure 3 (a), which as discussed in the next section, plots them for a specific case. They also illustrate the potential tradeoffs between line width $n_{L}$ and strip width $n_{S}$. Specifically, by increasing $n_{L}$ the line rate $\bar{R}_{n_{L}}^{L}$ decreases, though the fraction $\frac{n_{L}}{n_{S}+n_{L}}$ of pixels encoded at the higher rate increases, while increasing $n_{S}$ increases the fraction $\frac{n_{S}}{n_{L}+n_{S}}$ of pixels encoded at the lower rate, though the strip rate $\bar{R}_{n_{S}}^{S}$ increases.

In addition to considering the effect of $n_{S}$ and $n_{L}$ on rate, we can look at their influence on the rate redundancy $\Delta\left(n_{S}, n_{L}\right) \triangleq \frac{1}{|V|} D\left(X_{U}|| \tilde{X}_{U}\right)$, which is entirely due to encoding the lines independently and as moment-matching reduced MRFs. We use the shorthand notation $\tilde{X}_{B_{n_{L}}}$ to indicate the moment-matching reduced MRF on $B_{n_{L}}$ and $D\left(X_{B_{n_{L}}} \| \tilde{X}_{B_{n_{L}}}\right)$ to denote the divergence between the marginal and momentmatching reduced MRF distributions for $X_{B_{n_{L}}}$.

Proposition 5.5 The per-row rate redundancy due to coding $X_{U} \sim p\left(G ; X_{U} ; \theta\right)$ as a reduced $M R F X_{U} \sim p\left(G_{U} ; X_{U}: \theta_{U}^{*}\right)$ is

$$
\bar{\Delta}\left(n_{S}, n_{L}\right)=\frac{n_{S}}{n_{S}+n_{L}} I\left(X_{r_{1}} ; X_{r_{-n_{S}}}\right)+\frac{n_{L}}{n_{S}+n_{L}} D\left(X_{B_{n_{L}}} \| \tilde{X}_{B_{n_{L}}}\right),
$$

where $r_{1}$ is the 1 st row of a line, and $r_{-n_{S}}$ is the last row of the previous line. 
Proof To prove the proposition, consider a joint distribution $p\left(x_{1}, \ldots, x_{N}\right)$ on $N$ variables, where we have in mind each variable representing one of the $N=k+1$ lines. By approximating $p\left(x_{1}, \ldots, x_{N}\right)$ with $\tilde{p}\left(x_{1}, \ldots, x_{N}\right)=\prod_{i=1}^{N} \tilde{p}\left(x_{i}\right)$ we can see that the divergence between $p$ and $\tilde{p}$ is

$$
\begin{aligned}
D(p \| \tilde{p}) & =\sum_{x_{1}, \ldots, x_{N}} p\left(x_{1}, \ldots, x_{N}\right) \log \frac{p\left(x_{1}, \ldots, x_{N}\right)}{\tilde{p}\left(x_{1}\right) \cdots \tilde{p}\left(x_{N}\right)} \\
& =-\sum_{x_{1}, \ldots, x_{N}} p\left(x_{1}, \ldots, x_{N}\right) \log \tilde{p}\left(x_{1}\right) \cdots \tilde{p}\left(x_{N}\right)-H\left(X_{1}, \ldots, X_{N}\right) \\
& =\sum_{i=1}^{N} \sum_{x_{i}}-p\left(x_{i}\right) \log \tilde{p}\left(x_{i}\right)-H\left(X_{1}, \ldots, X_{N}\right) \\
& =\sum_{i=1}^{N}\left[H\left(X_{i}\right)+D\left(p\left(X_{i}\right) \| \tilde{p}\left(X_{i}\right)\right)\right]-H\left(X_{1}, \ldots, X_{N}\right) \\
& =\sum_{i=1}^{N}\left[H\left(X_{i}\right)-H\left(X_{i} \mid X_{i-1}, \ldots, X_{1}\right)+D\left(p\left(X_{i}\right) \| \tilde{p}\left(X_{i}\right)\right)\right] \\
& =\sum_{i=2}^{N} I\left(X_{i} ; X_{i-1}\right)+\sum_{i=1}^{N} D\left(p\left(X_{i}\right) \| \tilde{p}\left(X_{i}\right)\right) .
\end{aligned}
$$

Applying the stationarity assumption, weighting the last two terms by the (approximate) fractions in (3), and substituting $N=k+1$ and $X_{i}=X_{B_{n_{L}}}$ yields

$$
\bar{\Delta}\left(n_{S}, n_{L}\right)=\frac{n_{S}}{n_{S}+n_{L}} I\left(X_{B_{n_{L}}} ; X_{B_{n_{L}},-n_{S}}\right)+\frac{n_{L}}{n_{S}+n_{L}} D\left(X_{B_{n_{L}}} \| \tilde{X}_{B_{n_{L}}}\right),
$$

where $I\left(X_{B_{n_{L}}} ; X_{B_{n_{L}},-n_{S}}\right)$ is the mutual information between two $n_{L} \times N$ rectangular blocks of sites separated by a $n_{S} \times N$ rectangular block of sites. To finish the proof, it suffices to consider $I\left(X_{1}, X_{2} ; Y_{1}, Y_{2}\right)$ where $X_{1}-X_{2}-Y_{1}-Y_{2}$ form a Markov Chain. In this case,

$$
\begin{aligned}
I\left(X_{1}, X_{2} ; Y_{1}, Y_{2}\right) & =H\left(Y_{1}, Y_{2}\right)-H\left(Y_{1}, Y_{2} \mid X_{1}, X_{2}\right) \\
& =H\left(Y_{1}\right)+H\left(Y_{2} \mid Y_{1}\right)-H\left(Y_{1} \mid X_{1}, X_{2}\right)-H\left(Y_{2} \mid Y_{1}, X_{1}, X_{2}\right) \\
& =H\left(Y_{1}\right)+H\left(Y_{2} \mid Y_{1}\right)-H\left(Y_{1} \mid X_{2}\right)-H\left(Y_{2} \mid Y_{1}\right) \\
& =H\left(Y_{1}\right)-H\left(Y_{1} \mid X_{2}\right) \\
& =I\left(Y_{1} ; X_{2}\right) .
\end{aligned}
$$

Making the appropriate substitutions yields

$$
\bar{\Delta}\left(n_{S}, n_{L}\right)=\frac{n_{S}}{n_{S}+n_{L}} I\left(X_{r_{1}} ; X_{r_{-n_{S}}}\right)+\frac{n_{L}}{n_{S}+n_{L}} D\left(X_{B_{n_{L}}} \| \tilde{X}_{B_{n_{L}}}\right),
$$

where $I\left(X_{r_{1}} ; X_{{r-n_{S}}}\right)$ is the mutual information between the 1st row of a line and the last row of the previous line. This completes the proof. 
This proposition shows specifically how the redundancy of RCC has two components: a correlation redundancy $I\left(X_{r_{1}} ; X_{r_{-n_{S}}}\right)$ due to encoding the lines independently of one another, and a distribution redundancy $D\left(X_{B_{n_{L}}} \| \tilde{X}_{B_{n_{L}}}\right)$ due to approximating the lines as moment matching reduced MRFs.

Proposition 5.6 $I\left(X_{r_{1}} ; X_{r_{-n_{S}}}\right)$ is decreasing in $n_{S}$.

Proof We let $X_{r_{i, 1}}$ denote the 1st row of the $i$-th line and $X_{r_{i-1, n_{L}}}$ and $X_{r_{i-1, n_{L}-1}}$ denote, respectively, the $n_{L}$-th and $\left(n_{L}-1\right)$-st lines of the $(i-1)$-st line.

$$
\begin{aligned}
I\left(X_{r_{1}} ; X_{r_{-n} S}\right) & =H\left(G ; r_{i, 1} ; \theta\right)-H\left(G ; X_{r_{i, 1}} \mid X_{r_{i-1, n_{L}}} ; \theta\right) \\
& =H\left(G ; r_{i, 1} ; \theta\right)-H\left(G ; X_{r_{i, 1}} \mid X_{r_{i-1, n_{L}}}, X_{r_{i-1, n_{L}-1}} ; \theta\right) \\
& >H\left(G ; r_{i, 1} ; \theta\right)-H\left(G ; X_{r_{i, 1}} \mid X_{r_{i-1, n_{L}-1}} ; \theta\right) \\
& =I\left(X_{r_{1}} ; X_{r_{-\left(n_{S}+1\right)}}\right),
\end{aligned}
$$

where (11) is due to the Markov property and $(12)$ is due to removing conditioning. This completes the proof.

To analyze the distribution redundancy, we let $\tilde{\tilde{X}}_{B_{n}}$ be the marginal distribution of $X_{B_{n-1}}$ as a subset of the moment-matching reduced MRF $\tilde{X}_{B_{n}}$ on $B_{n}$. More generally, $X_{B_{n}}$ decorated with $k$ "tildes" indicates the marginal distribution of $X_{B_{n-k+1}}$ as a subset of the moment-matching reduced MRF $\tilde{X}_{B_{n}}$ on $B_{n}$. Moreover, we let $\theta_{n}^{*}$ be shorthand for $\theta_{B_{n}}^{*}$. We then have the following recursive expression for the distribution redundancy.

\section{Proposition 5.7}

$$
\begin{aligned}
D\left(X_{B_{n_{L}}} \| \tilde{X}_{B_{n_{L}}}\right)= & D\left(X_{B_{n_{L}-1}} \| \tilde{X}_{B_{n_{L}-1}}\right)-D\left(\tilde{\tilde{X}}_{B_{n_{L}}}|| \tilde{X}_{B_{n_{L}-1}}\right) \\
& +H\left(G_{B_{n_{L}}} ; r_{n_{L}} \mid r_{n_{L}-1} ; \theta_{n_{L}}^{*}\right)-H\left(G ; r_{n_{L}} \mid r_{n_{L}-1} ; \theta\right),
\end{aligned}
$$

where $D\left(\tilde{\tilde{X}}_{B_{n_{L}}} \| \tilde{X}_{B_{n_{L}-1}}\right)$ is the divergence between the marginal distribution of $X_{B_{n_{L}-1}}$ as a subfield of $\tilde{X}_{B_{n_{L}}}$ and the reduced $M R F \tilde{X}_{B_{n_{L}-1}}$ on $B_{n_{L}-1}$, and where $H\left(\cdot ; r_{n} \mid r_{n-1} ; \cdot\right)$ is the conditional entropy of row $r_{n}$ condition on row $r_{n-1}$ for the specified graph and parameter vector.

Proof We prove the proposition by using the fact that the divergence $D\left(X_{B_{n_{L}}} \| \tilde{X}_{B_{n_{L}}}\right)$ between the marginal distribution of $X_{B_{n_{L}}}$ and the reduced MRF for $X_{B_{n_{L}}}$ can be 
expressed as the difference between the entropy of the latter and that of the former. Specifically,

$$
\begin{aligned}
D\left(X_{B_{n_{L}}} \| \tilde{X}_{B_{n_{L}}}\right)= & H\left(G_{B_{n_{L}}} ; X_{B_{n_{L}}} ; \theta_{n_{L}}^{*}\right)-H\left(G ; X_{B_{n_{L}}} ; \theta\right) \\
= & H\left(G_{B_{n_{L}}} ; X_{B_{n_{L}-1}} ; \theta_{n_{L}}^{*}\right)-H\left(G ; X_{B_{n_{L}-1}} ; \theta\right) \\
& +H\left(G_{B_{n_{L}}} ; r_{n_{L}} \mid r_{n_{L}-1} ; \theta_{n_{L}}^{*}\right)-H\left(G ; r_{n_{L}} \mid r_{n_{L}-1} ; \theta\right) \\
= & H\left(G_{B_{n_{L}-1}} ; X_{B_{n_{L}-1}} ; \theta_{n_{L}-1}^{*}\right)-D\left(\tilde{X}_{B_{n_{L}}} \| \tilde{X}_{B_{n_{L}-1}}\right) \\
& -H\left(G ; X_{B_{n_{L}-1}} ; \theta\right)+H\left(G_{B_{n_{L}}} ; r_{n_{L}} \mid r_{n_{L}-1} ; \theta_{n_{L}}^{*}\right)-H\left(G ; r_{n_{L}} \mid r_{n_{L}-1} ; \theta\right) \\
= & H\left(G_{B_{n_{L}-1}} ; X_{B_{n_{L}-1}} ; \theta_{n_{L}-1}^{*}\right)-H\left(G ; X_{B_{n_{L}-1}} ; \theta\right) \\
& -D\left(\tilde{\tilde{X}}_{B_{n_{L}}} \| \tilde{X}_{B_{n_{L}-1}}\right)+H\left(G_{B_{n_{L}}} ; r_{n_{L}} \mid r_{n_{L}-1} ; \theta_{n_{L}}^{*}\right)-H\left(G ; r_{n_{L}} \mid r_{n_{L}-1} ; \theta\right) \\
= & D\left(X_{B_{n_{L}-1}} \| \tilde{X}_{B_{n_{L}-1}}\right)-D\left(\tilde{\tilde{X}}_{B_{n_{L}}} \| \tilde{X}_{B_{n_{L}-1}}\right) \\
& +H\left(G_{B_{n_{L}}} ; r_{n_{L}} \mid r_{n_{L}-1} ; \theta_{n_{L}}^{*}\right)-H\left(G ; r_{n_{L}} \mid r_{n_{L}-1} ; \theta\right) .
\end{aligned}
$$

This completes the proof.

Furthermore, the divergence $D\left(\tilde{\tilde{X}}_{B_{n_{L}-1}} \| \tilde{X}_{B_{n_{L}-1}}\right)$ has the following recursive relationship.

\section{Proposition 5.8}

$$
\begin{aligned}
D\left(\tilde{\tilde{X}}_{B_{n-k+1}} \| \tilde{X}_{B_{n-k}}\right)= & D\left(\tilde{\tilde{\tilde{X}}}_{B_{n-k+1}} \| \tilde{X}_{B_{n-k-1}}\right)-D\left(\tilde{\tilde{X}}_{B_{n-k}} \| \tilde{X}_{B_{n-k-1}}\right) \\
& +H\left(G_{B_{n-k}} ; r_{n-k} \mid r_{n-k-1} ; \theta_{n-k}^{*}\right)-H\left(G_{B_{n-k+1}} ; r_{n-k} \mid r_{n-k-1} ; \theta_{n-k+1}^{*}\right)
\end{aligned}
$$

where $D\left(\tilde{\tilde{\tilde{X}}}_{B_{n-k+1}} \| \tilde{X}_{B_{n-k-1}}\right)$ is the divergence between the marginal distribution of $X_{B_{n-k-1}}$ as a subfield of $\tilde{X}_{B_{n-k+1}}$ and the reduced $M R F \tilde{X}_{B_{n-k-1}}$ on $B_{n-k-1}$.

\section{Proof}

$$
\begin{aligned}
D\left(\tilde{\tilde{X}}_{B_{n-k+1}} \| \tilde{X}_{B_{n-k}}\right)= & H\left(G_{B_{n-k}} ; X_{B_{n-k}} ; \theta_{n-k}^{*}\right)-H\left(G_{B_{n-k+1}} ; X_{B_{n-k}} ; \theta_{n-k+1}^{*}\right) \\
= & H\left(G_{B_{n-k}} ; X_{B_{n-k-1}} ; \theta_{n-k}^{*}\right)-H\left(G_{B_{n-k+1}} ; X_{B_{n-k-1}} ; \theta_{n-k+1}^{*}\right) \\
& +H\left(G_{B_{n-k}} ; r_{n-k} \mid r_{n-k-1} ; \theta_{n-k}^{*}\right)-H\left(G_{B_{n-k+1}} ; r_{n-k} \mid r_{n-k-1} ; \theta_{n-k+1}^{*}\right) \\
= & H\left(G_{B_{n-k-1}} ; X_{B_{n-k-1}} ; \theta_{n-k-1}^{*}\right)-D\left(\tilde{\tilde{X}}_{n-k} \| \tilde{X}_{n-k-1}\right) \\
& -H\left(G_{B_{n-k+1}} ; X_{B_{n-k-1}} ; \theta_{n-k+1}^{*}\right)+H\left(G_{B_{n-k}} ; r_{n-k} \mid r_{n-k-1} ; \theta_{n-k}^{*}\right) \\
& -H\left(G_{B_{n-k+1}} ; r_{n-k} \mid r_{n-k-1} ; \theta_{n-k+1}^{*}\right) \\
= & D\left(\tilde{\tilde{X}}_{n-k+1} \| \tilde{X}_{n-k-1}\right)-D\left(\tilde{\tilde{X}}_{n-k}|| \tilde{X}_{n-k-1}\right) \\
& +H\left(G_{B_{n-k}} ; r_{n-k} \mid r_{n-k-1} ; \theta_{n-k}^{*}\right)-H\left(G_{B_{n-k+1}} ; r_{n-k} \mid r_{n-k-1} ; \theta_{n-k+1}^{*}\right) .
\end{aligned}
$$

This completes the proof. 


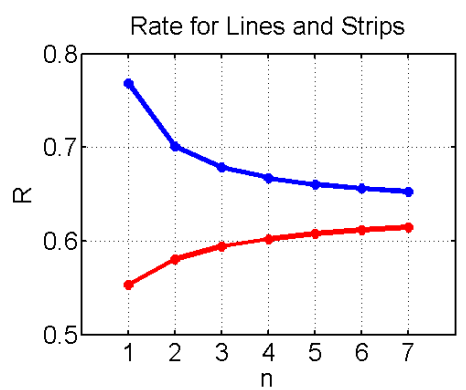

(a)

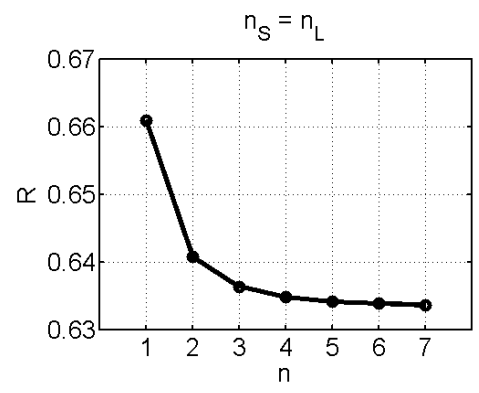

(c)

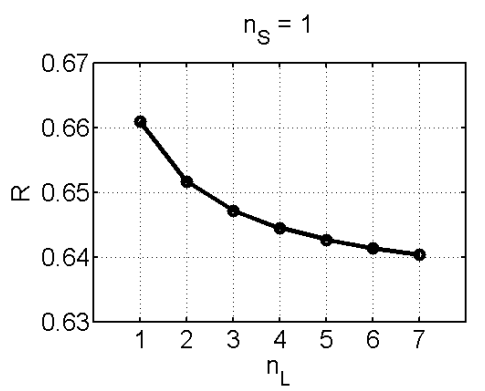

(b)

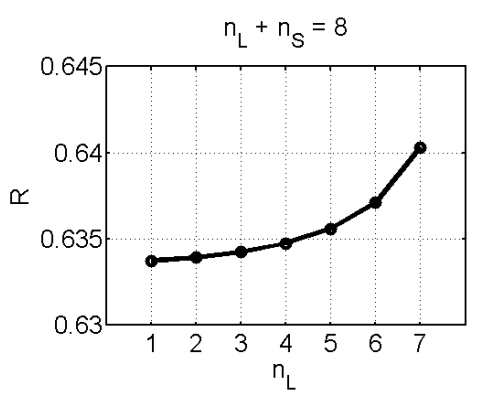

(d)

Figure 3: Rate (a) for lines (blue) and strips (red); (b) as a function of $n_{L}$ for $n_{S}=1$; (c) as a function of $n=n_{S}=n_{L}$; and (d) for $n_{S}+n_{L}=8$.

Intuitively we would expect the term $D\left(X_{B_{n_{L}}} \| \tilde{X}_{B_{n_{L}}}\right)$ to decrease in $n_{L}$, as this divergence is zero when $n_{L}=M$ and indeed we conjecture that this is the case. At the very least, we expect $\frac{1}{n_{L}} D\left(X_{B_{n_{L}}} \| \tilde{X}_{B_{n_{L}}}\right)$ to decrease in $n_{L}$.

We now consider the effects of changing $n_{S}$ and $n_{L}$ on redundancy, as expressed in Proposition 5.5. Increasing $n_{S}$ decreases distribution redundancy through the factor $\frac{n_{L}}{n_{S}+n_{L}}$. It is not so clear what happens to the correlation redundancy, as increasing $n_{S}$ increases the fraction $\frac{n_{S}}{n_{S}+n_{L}}$, while decreasing the information $I\left(X_{r_{1}} ; X_{r_{-n_{S}}}\right)$. However, if we keep $n_{S}$ and $n_{L}$ proportional to one another, as $n_{S}$ increases, the fraction stays the same, the correlation redundancy decreases, and assuming the conjecture, so too does distribution redundancy.

Similarly, increasing $n_{L}$ decreases the correlation redundancy through the factor $\frac{n_{S}}{n_{S}+n_{L}}$. Even assuming the above conjecture, it is not clear what happens to the distribution redundancy, as increasing $n_{L}$ increases the fraction $\frac{n_{L}}{n_{S}+n_{L}}$, while decreasing the divergence $D\left(X_{B_{n_{L}}} \| \tilde{X}_{B_{n_{L}}}\right)$. However, as mentioned above, if $n_{S}$ and $n_{L}$ increase proportionally to one another, then the fraction stays the same and both the correlation and distribution redundancies decrease in $n_{L}$.

The complexity of this coding scheme can be expressed as

$$
C_{n_{S}, n_{L}}=\frac{n_{S}}{n_{S}+n_{L}}|\mathcal{X}|^{n_{S}} c_{S}+\frac{n_{L}}{n_{S}+n_{L}}|\mathcal{X}|^{n_{L}} c_{L}
$$

where $|\mathcal{X}|$ denotes the number of elements of $\mathcal{X}$, and $c_{S}$ and $c_{L}$ are factors relating the complexity of encoding a strip versus a line. For example, numerical simulations show that for $n_{S}=n_{L}$, the run-time involved in encoding a strip is a little higher 
than that for a line, which is due to additional operations for conditioning on the boundary of a strip. However, the difference becomes negligible as $n_{S}$ and $n_{L}$ become larger. As a result, the complexity $C_{n_{S}, n_{L}}$ is dominated by $\max \left\{n_{S}, n_{L}\right\}$. Given a constraint $\max \left\{n_{S}, n_{L}\right\} \leq n^{*}$ on the maximum exponent in the complexity, since both Proposition 5.6 and our conjecture indicate choosing $n_{S}$ and $n_{L}$ each to be as large as possible, we propose setting $n_{S}=n_{L}$.

\section{Example: Homogeneous Ising Model}

We simulated a homogeneous Ising model with edge parameter $\theta_{i j}=0.4$ and node parameter $\theta_{i}=0$ using Gibbs sampling. To encode the lines with line width $n_{L}$, we approximate the moment-matching parameter $\theta_{n_{L}}^{*}$ by minimizing the empirical cross entropy

$$
H_{n_{L}}^{n K}\left(\tilde{\theta}_{n_{L}}\right)=\frac{1}{n K} \sum_{L_{i}} \sum_{j=1}^{n}-\log p\left(G_{L_{i}} ; \mathbf{x}_{L_{i}}^{(j)} ; \tilde{\theta}_{n_{L}}\right) .
$$

Note that even for a homogeneous MRF, the moment-matching parameter for a subset $U$ will in general not be homogeneous.

The line rate $\bar{R}_{n_{L}}^{L}$ is approximated by

$$
\hat{R}_{n_{L}}^{L}=\frac{1}{n K} \sum_{L_{i}} \sum_{j=1}^{n}-\log p\left(G_{L_{i}} ; \mathbf{x}_{L_{i}}^{(j)} ; \theta_{n_{L}}^{*}\right) .
$$

Similarly, $\bar{R}_{n_{S}}^{S}$ is approximated by

$$
\hat{R}_{n_{S}}^{S}=\frac{1}{n K} \sum_{S_{i}} \sum_{j=1}^{n}-\log p\left(G ; \mathbf{x}_{S_{i}}^{(j)} \mid \mathbf{x}_{\partial S_{i}}^{(j)} ; \theta\right) .
$$

Figure 3(a) shows $\hat{R}_{n_{L}}^{L}$ and $\hat{R}_{n_{S}}^{L}$. As predicted by Propositions 5.1, 5.3, and 5.4, $\hat{R}_{n_{S}}^{S}$ is increasing in $n_{S}, \hat{R}_{n_{L}}^{L}$ is decreasing in $n_{L}$, and $\hat{R}_{n_{S}}^{S}<\hat{R}_{n_{L}}^{L}$ for all $n_{S}, n_{L}$. We computed $\hat{R}_{n_{S}, n_{L}}$ from $\hat{R}_{n_{L}}^{L}$ and $\hat{R}_{n_{S}}^{L}$ using (3), and as seen in Figure 3(b), we found that $\hat{R}_{n_{S}, n_{L}}$ decreases as $n_{L}$ increases for constant $n_{S}$. We also found, see Figure 3 (c), that $\hat{R}_{n_{S}, n_{L}}$ decreases with $n$ increasing when $n=n_{L}=n_{S}$, which is consistent with the earlier discussion that presumed the conjecture. Finally, we found that if one holds the sum $n_{L}+n_{S}$ constant, then the rate $\hat{R}_{n_{S}, n_{L}}$ is minimized when $n_{L}=1$. This indicates that the information $I\left(X_{r_{1}} ; X_{r_{-n_{S}}}\right)$ decreases with $n_{S}$ faster than the divergence $D\left(X_{B_{n_{L}}} \| \tilde{X}_{B_{n_{L}}}\right)$ decreases with $n_{L}$. Though not apparent in the Figure, we found that $\hat{R}_{7,7}<\hat{R}_{7,1}$, an improvement over our earlier paper [10] which focused exclusively on $n_{L}=1$. However, the improvement is nominal, so therefore, at least for this particular value of $\theta_{i j}$, does not justify the significantly increased complexity. 


\section{Concluding Remarks}

In this paper we have addressed the topic of tradeoffs in the choice of the width $n_{L}$ and spacing $n_{S}$ of the cutset components in Reduced Cutset Coding of Markov random fields. We have provided analysis from the perspective of the rate of this scheme in terms of the rates for encoding lines and strips and the relative contributions of each to the overall rate. We have shown that the rate for encoding lines with the moment-matching reduced MRF decreases with $n_{L}$, and that the rate for encoding strips increases with $n_{S}$, and on the basis of just these results one might conclude that large $n_{L}$ and small $n_{S}$ would provide an optimal combination. However, we also show that for all combinations of $n_{L}$ and $n_{S}$, the rate for encoding lines is strictly greater than the rate for encoding strips. Moreover, the fraction $\frac{n_{L}}{n_{S}+n_{L_{n}}}$ of sites encoded at

the larger rate obviously increases with $n_{L}$, while the fraction $\frac{n_{L} n_{S}}{n_{S}+n_{L}}$ of sites encoded at the smaller rate obviously decreases with $n_{S}$.

Additionally, we have analyzed the problem from the perspective of the redundancy in the code, showing that this redundancy decomposes into a distribution redundancy due to approximating the lines as moment-matching reduced MRFs, and a correlation redundancy due to independent coding of the lines. We show that the correlation redundancy is decreasing in $n_{S}$ and provide analysis of the distribution redundancy and conjecture that it is decreasing in $n_{L}$. Indeed, numerical experiments with an Ising model corroborate this conjecture. Moreover, if we let $n_{L}$ be the height of the original image, then clearly the divergence $D\left(X_{B_{n_{L}}}|| \tilde{X}_{B_{n_{L}}}\right)=0$, and at least offhand, there is no reason to suspect that this divergence is non-monotonic in $n_{L}$. Naturally, though, further analysis of $D\left(X_{B_{n_{L}}} \| \tilde{X}_{B_{n_{L}}}\right)$ remain to be done, and at least at the moment, we suspect that the recursive relations for $D\left(X_{B_{n_{L}}} \| \tilde{X}_{B_{n_{L}}}\right)$ will be useful in proving our conjecture.

While for general row-invariant statistics $t$ and exponential parameters $\theta$ it is not clear what the best choices of $n_{L}$ and $n_{S}$ should be, our numerical experiments with a uniform Ising model with parameters $\theta_{i j}=0.4, \theta_{i}=0$ suggest that letting $n_{S}$ and $n_{L}$ both be as large as possible achieves a lower rate. However, since the decrease in rate over a large $n_{S}$ and $n_{L}=1$ is in the fourth decimal place (in terms of per-site rate), the greatly increased complexity in encoding lines with large $n_{L}$ does not seem worth it. However, more work remains to be done in understanding how differences in parameter values affect these tradeoffs. And more generally, beyond the Ising model, we would like to understand how the apparent tradeoffs between $n_{S}$ and $n_{L}$ vary with $\theta$ for different types of statistic $t$. Previous work of the authors [9, 11, 12] has looked at the relationship between positively correlated statistics $t$ and quantities of interest and it will be interesting to see if such statistics can be shown to have significant consequences for RCC.

\section{References}

[1] S. Amari and H. Nagaoka, Methods of Information Geometry, Oxford University Press, 2000.

[2] D. Anastassiou and D.J. Sakrison, "Some Results Regarding the Entropy Rate of Random Fields," IEEE Trans. Inform. Thy., vol. IT-28, pp. 340-343, Mar. 1982. 
[3] S. Boyd and L. Vandenberghe, Convex Optimization, Cambridge University Press, 2004.

[4] T. Cover and J. Thomas, Elements of Information Theory, Wiley, 2005.

[5] H.O. Georgii, "Gibbs Measures and Phase Transitions," De Gruyter, New York, 1988.

[6] G. Grimmett and D. Stirzaker, "Probability and Random Processes," Oxford, 2001.

[7] I. Kontoyiannis, "Pattern Matching and Lossy Data Compression on Random Fields," IEEE Tr. Inform. Thy., v. 49, pp. 1047-1051, April 2003.

[8] M.G. Reyes and D.L. Neuhoff, Arithmetic Compression of Markov Random Fields, Seoul, Korea, ISIT 2009.

[9] M. G. Reyes and D. L. Neuhoff, "Entropy Bounds for a Markov Random Subfield," Proc. ISIT, Seoul, Korea, pp. 309-313, July 2009.

[10] M.G. Reyes and D.L. Neuhoff, Lossless Reduced Cutset Coding of Markov Random Fields, Snowbird, UT, DCC 2010.

[11] M.G. Reyes, Cutset Based Processing and Compression of Markov Random Fields, Ph.D. thesis, University of Michigan, April 2011.

[12] M.G. Reyes, "Covariance and Entropy in Markov Random Fields," ITA, San Diego, 2013.

[13] M. G. Reyes, D. L. Neuhoff, T. N. Pappas, "Lossy Cutset Coding of Bilevel Images Based on Markov Random Fields," IEEE Trans. Img. Proc., vol. 23, pp. 1652-1665, April 2014.

[14] M. J. Wainwright and M. I. Jordan, Graphical models, exponential families and variational inference, Berkeley Tech. Report 649, Sept. 2003.

[15] I. H. Whitten, R. M. Neal, and J. G. Cleary, Arithmetic Coding For Data Compression, Comm. of the ACM, vol. 30, pp. 520-540, June 1987. 